\title{
Discovery of an Orally Bioavailable Gonadotropin-releasing Hormone (GnRH) Receptor Antagonist
}

Seon-Mi Kim, ${ }^{\dagger}$ Minhee Lee, ${ }^{\dagger}$ So Young Lee, ${ }^{\dagger}$ Euisun Park,${ }^{\star}$ Soo-Min Lee, ${ }^{\dagger}$ Eun Jeong Kim, ${ }^{\dagger}$ Min Young Han, ${ }^{\dagger}$ Taekyung, Yoo, ${ }^{\dagger}$ Jihyae Ann,${ }^{\S}$ Suyoung Yoon,${ }^{\S}$ Jiyoun Lee, ${ }^{\|}$and Jeewoo Lee* ${ }^{\S}$

\section{HPLC Conditions}

Column : Shiseido Capcell PAK C18 (5 $\mu \mathrm{m}, 4.6$ x $150 \mathrm{~mm})$

Conditions: Wavelength : $272 \mathrm{~nm}, 254 \mathrm{~nm}$

Temperature : $30{ }^{\circ} \mathrm{C}$

Flow rate : $1.00 \mathrm{~mL} / \mathrm{min}$ (gradient)

\begin{tabular}{|c|c|}
\hline \multicolumn{2}{|c|}{ Method A } \\
\hline Eluent & $\begin{array}{l}\text { a: } \mathrm{H}_{2} \mathrm{O} \text { containing } 0.1 \% \text { TFA } \\
\text { b: } \mathrm{MeCN}\end{array}$ \\
\hline Time(min) & $\%$ of eluent $\mathrm{b}$ \\
\hline 0 & 20 \\
\hline 1 & 20 \\
\hline 27 & 70 \\
\hline 30 & 95 \\
\hline 31 & 95 \\
\hline 31.5 & 20 \\
\hline 35 & 20 \\
\hline \multicolumn{2}{|c|}{ Method B } \\
\hline Eluent & $\begin{array}{l}\text { a: } \mathrm{H}_{2} \mathrm{O} \text { containing } 0.78 \% \mathrm{NH}_{4} \mathrm{OAc} \\
\text { b: } \mathrm{MeCN}\end{array}$ \\
\hline Time(min) & $\%$ of eluent $b$ \\
\hline 0 & 45 \\
\hline 1 & 45 \\
\hline 30 & 95 \\
\hline 31 & 95 \\
\hline 31.5 & 45 \\
\hline 35 & 45 \\
\hline \multicolumn{2}{|r|}{ Method C } \\
\hline Eluent & $\begin{array}{l}\text { a: } \mathrm{H}_{2} \mathrm{O} \text { containing } 0.78 \% \mathrm{NH}_{4} \mathrm{OAc} \\
\text { b: } \mathrm{MeCN}\end{array}$ \\
\hline Time(min) & $\%$ of eluent $\mathrm{b}$ \\
\hline 0 & 35 \\
\hline 1 & 35 \\
\hline 30 & 90 \\
\hline 31 & 90 \\
\hline 31.5 & 35 \\
\hline 35 & 35 \\
\hline
\end{tabular}




\section{Purity}

\begin{tabular}{|c|c|c|c|}
\hline Compound & Method & $\begin{array}{l}\text { Retention time } \\
(\text { min) }\end{array}$ & $\begin{array}{c}\text { Purity } \\
(\%)\end{array}$ \\
\hline $4 \mathbf{a}$ & $\mathrm{C}$ & 3.99 & 95.74 \\
\hline $4 b$ & A & 11.31 & 96.20 \\
\hline $4 c$ & $\mathrm{C}$ & 13.39 & 99.39 \\
\hline 4d & $\mathrm{A}$ & 10.78 & 99.33 \\
\hline $4 e$ & $\mathrm{~B}$ & 10.57 & 98.72 \\
\hline $4 f$ & $\mathrm{~A}$ & 14.36 & 95.90 \\
\hline $4 \mathrm{~g}$ & A & 18.17 & 96.00 \\
\hline $4 h$ & $\mathrm{~A}$ & 8.64 & 98.34 \\
\hline $4 i$ & $\mathrm{~A}$ & 9.55 & 99.01 \\
\hline $4 \mathbf{j}$ & $\mathrm{A}$ & 9.66 & 99.47 \\
\hline $4 \mathbf{k}$ & $\mathrm{C}$ & 4.86 & 96.85 \\
\hline 41 & $\mathrm{~A}$ & 17.35 & 99.30 \\
\hline $4 m$ & $\mathrm{~A}$ & 18.50 & 95.41 \\
\hline $4 n$ & $\mathrm{~A}$ & 19.14 & 99.33 \\
\hline 40 & $\mathrm{~A}$ & 19.16 & 99.02 \\
\hline $4 p$ & $\mathrm{C}$ & 20.75 & 99.58 \\
\hline $4 q$ & $\mathrm{~B}$ & 12.05 & 97.85 \\
\hline $4 r$ & $\mathrm{~A}$ & 23.45 & 97.64 \\
\hline $4 s$ & $\mathrm{~A}$ & 10.01 & 96.52 \\
\hline $4 t$ & $\mathrm{C}$ & 19.41 & 96.94 \\
\hline $4 u$ & $\mathrm{C}$ & 21.13 & 98.84 \\
\hline $10 \mathbf{a}$ & $\mathrm{C}$ & 9.13 & 98.48 \\
\hline $10 b$ & $\mathrm{C}$ & 9.07 & 95.91 \\
\hline $10 \mathrm{c}$ & $\mathrm{C}$ & 9.57 & 98.04 \\
\hline 10d & $\mathrm{A}$ & 16.50 & 99.65 \\
\hline $10 \mathrm{e}$ & A & 10.49 & 96.39 \\
\hline $10 f$ & $\mathrm{~A}$ & 11.50 & 96.34 \\
\hline $10 \mathrm{~g}$ & $\mathrm{C}$ & 15.52 & 95.10 \\
\hline 10h & $\mathrm{A}$ & 10.86 & 99.22 \\
\hline 11a & $\mathrm{A}$ & 8.58 & 95.41 \\
\hline $11 \mathrm{~b}$ & A & 10.17 & 97.56 \\
\hline 11c & A & 9.65 & 95.35 \\
\hline 11d & $\mathrm{A}$ & 8.98 & 98.40 \\
\hline 11e & A & 10.12 & 98.97 \\
\hline $11 f$ & $\mathrm{~A}$ & 10.48 & 98.12 \\
\hline $11 \mathrm{~g}$ & $A$ & 11.44 & 99.16 \\
\hline $11 \mathrm{~h}$ & $\mathrm{~A}$ & 12.06 & 97.78 \\
\hline $11 \mathrm{i}$ & A & 9.82 & 99.38 \\
\hline $\mathbf{1 1 j}$ & A & 10.63 & 98.17 \\
\hline $11 \mathrm{k}$ & $\mathrm{A}$ & 9.81 & 95.78 \\
\hline 111 & $\mathrm{C}$ & 14.42 & 97.22 \\
\hline $11 \mathrm{~m}$ & $A$ & 9.96 & 97.17 \\
\hline 11n & $\mathrm{C}$ & 18.81 & 98.43 \\
\hline 110 & $\mathrm{~A}$ & 11.84 & 98.19 \\
\hline 11p & $\mathrm{A}$ & 12.49 & 96.20 \\
\hline $11 q$ & A & 9.53 & 98.04 \\
\hline 11r & A & 10.89 & 97.91 \\
\hline
\end{tabular}




\begin{tabular}{|c|c|c|c|}
\hline $11 \mathrm{~s}$ & A & 9.69 & 99.19 \\
\hline $11 t$ & A & 8.79 & 97.28 \\
\hline 11u & A & 9.92 & 97.65 \\
\hline $11 v$ & A & 11.17 & 95.27 \\
\hline $11 w$ & A & 12.14 & 96.50 \\
\hline $11 x$ & $\mathrm{C}$ & 20.44 & 99.07 \\
\hline $11 y$ & $\mathrm{C}$ & 16.89 & 97.25 \\
\hline $11 z$ & $\mathrm{C}$ & 19.92 & 99.54 \\
\hline $12 a$ & B & 14.05 & 99.28 \\
\hline $12 b$ & B & 13.13 & 99.02 \\
\hline $12 \mathrm{c}$ & B & 14.57 & 98.59 \\
\hline 12d & B & 10.47 & 97.08 \\
\hline $12 \mathrm{e}$ & $\mathrm{C}$ & 23.01 & 95.07 \\
\hline $12 \mathrm{f}$ & B & 14.40 & 95.54 \\
\hline $12 \mathrm{~g}$ & B & 12.42 & 99.40 \\
\hline $12 \mathrm{~h}$ & $\mathrm{C}$ & 22.37 & 96.32 \\
\hline $12 \mathrm{i}$ & B & 13.48 & 99.06 \\
\hline $12 \mathrm{j}$ & B & 6.09 & 95.81 \\
\hline $12 \mathrm{k}$ & $\mathrm{C}$ & 11.15 & 98.19 \\
\hline 12I & $\mathrm{C}$ & 12.60 & 96.49 \\
\hline $12 \mathrm{~m}$ & $\mathrm{C}$ & 15.85 & 95.02 \\
\hline 12n & $\mathrm{C}$ & 10.78 & 96.52 \\
\hline 120 & $\mathrm{C}$ & 13.73 & 95.64 \\
\hline $12 p$ & B & 2.39 & 98.08 \\
\hline $12 q$ & B & 12.63 & 99.40 \\
\hline $12 r$ & B & 15.11 & 99.52 \\
\hline $12 \mathrm{~s}$ & $\mathrm{C}$ & 18.19 & 97.25 \\
\hline $12 t$ & $\mathrm{C}$ & 17.39 & 97.62 \\
\hline $12 u$ & $\mathrm{C}$ & 18.57 & 98.18 \\
\hline $12 \mathrm{v}$ & $\mathrm{C}$ & 10.55 & 95.01 \\
\hline $12 w$ & $\mathrm{C}$ & 17.13 & 97.10 \\
\hline $13 a$ & B & 6.82 & 95.62 \\
\hline 13b & B & 9.43 & 95.94 \\
\hline $13 c$ & B & 7.73 & 95.96 \\
\hline 13d & $\mathrm{C}$ & 12.99 & 96.45 \\
\hline 13e & B & 9.40 & 97.74 \\
\hline $13 f$ & B & 9.10 & 98.11 \\
\hline 13g & B & 12.10 & 98.33 \\
\hline $13 \mathrm{~h}$ & B & 11.10 & 98.19 \\
\hline $13 i$ & $\mathrm{C}$ & 19.59 & 96.83 \\
\hline 13j & $\mathrm{C}$ & 17.49 & 95.21 \\
\hline 13k & $\mathrm{C}$ & 18.50 & 97.58 \\
\hline 13I & $\mathrm{C}$ & 17.57 & 96.27 \\
\hline $13 \mathrm{~m}$ & B & 5.98 & 99.59 \\
\hline 13n & $\mathrm{C}$ & 9.93 & 98.76 \\
\hline 130 & $\mathrm{C}$ & 10.95 & 95.20 \\
\hline $13 p$ & B & 3.57 & 96.95 \\
\hline $13 q$ & B & 3.49 & 98.86 \\
\hline $13 r$ & B & 20.44 & 95.40 \\
\hline $13 s$ & $\mathrm{~B}$ & 13.96 & 97.65 \\
\hline
\end{tabular}




\begin{tabular}{|c|c|c|c|}
\hline $13 t$ & C & 15.62 & 95.94 \\
\hline $13 u$ & $\mathrm{C}$ & 19.87 & 97.34 \\
\hline $13 v$ & B & 9.67 & 95.30 \\
\hline $13 w$ & B & 14.36 & 98.08 \\
\hline $14 a$ & B & 8.32 & 98.68 \\
\hline $14 b$ & B & 6.77 & 98.89 \\
\hline $14 c$ & B & 7.18 & 98.41 \\
\hline 14d & B & 7.52 & 98.99 \\
\hline $14 \mathrm{e}$ & B & 5.82 & 95.10 \\
\hline $14 f$ & B & 11.09 & 97.97 \\
\hline $14 \mathrm{~g}$ & B & 9.24 & 98.37 \\
\hline $14 \mathrm{~h}$ & B & 13.11 & 98.19 \\
\hline $14 i$ & $\mathrm{C}$ & 16.34 & 95.83 \\
\hline $14 j$ & $\mathrm{C}$ & 22.52 & 95.56 \\
\hline $14 k$ & B & 14.52 & 96.83 \\
\hline 141 & B & 9.85 & 95.15 \\
\hline $14 \mathrm{~m}$ & $\mathrm{C}$ & 14.41 & 95.82 \\
\hline $14 n$ & $\mathrm{C}$ & 15.04 & 95.23 \\
\hline 140 & $\mathrm{C}$ & 8.95 & 97.07 \\
\hline $14 p$ & $\mathrm{C}$ & 18.73 & 97.93 \\
\hline $14 q$ & $\mathrm{C}$ & 21.92 & 95.98 \\
\hline $14 r$ & $\mathrm{C}$ & 22.41 & 95.57 \\
\hline $14 s$ & $\mathrm{C}$ & 15.27 & 95.30 \\
\hline $17 a$ & $\mathrm{C}$ & 5.36 & 97.34 \\
\hline $17 \mathrm{~b}$ & $\mathrm{C}$ & 5.52 & 96.65 \\
\hline $17 \mathrm{c}$ & $\mathrm{C}$ & 5.84 & 96.25 \\
\hline 18a & $\mathrm{C}$ & 13.81 & 99.01 \\
\hline $18 b$ & $\mathrm{C}$ & 12.88 & 97.27 \\
\hline $18 \mathrm{c}$ & $\mathrm{C}$ & 15.90 & 97.26 \\
\hline 19a & B & 8.38 & 95.65 \\
\hline 19b & $\mathrm{C}$ & 11.47 & 98.98 \\
\hline 20 & $\mathrm{C}$ & 13.07 & 97.20 \\
\hline 26 & $\mathrm{C}$ & 18.56 & 98.90 \\
\hline $28 a$ & $\mathrm{C}$ & 11.88 & 97.03 \\
\hline $28 b$ & $\mathrm{C}$ & 11.64 & 96.54 \\
\hline
\end{tabular}

